\title{
BOUNDARY VALUE PROBLEMS FOR PARTIAL DIFFERENTIAL EQUATIONS WITH PIECEWISE CONSTANT DELAY
}

\author{
JOSEPH WIENER \\ Department of Mathematics \\ The University of Texas - Pan American \\ Edinburg, Texas 78539 \\ (Recetved February 21, 1990)
}

ABSTRACT. The influence of certain discontinuous delays on the behavior of solutions to some typical equations of mathematical physics is studied.

KEY WORDS AND PHRASES. Partial Differential Equation, Piecewise Constant Delay, Boundary Value Problem, Fourier Method, Positive Operator, Weak solution.

1980 AMS Subject Classification Codes. 35A05, 35B25, 35L10, 34K25.

\section{INTRODUCTION.}

Functional differential equations(FDE) with delay provide a mathematical model for a physical or biological system in which the rate of change of the system depends upon its past history. The theory of FDE with continuous argument is well developed and has numerous applications in natural and engineering sciences. This' note continues our earlier work [1-5] in an attempt to extend this theory to differential equations with discontinuous argument deviations. In these papers, ordinary differential equations with arguments having intervals of constancy have been studied. Such equations represent a hybrid of continuous and discrete dynamical systems and combine properties of both differential and difference equations. They include as particular cases loaded and impulse equations, hence their importance in control theory and in certain biomedical models. Continuity of a solution at a point joining any two consecutive intervals implies recursion relations for the values of the solution at such points. Therefore, differential equations with piecewise constant argrument (EPCA) are intrinsically closer to difference rather than differential 
equations. Here boundary value problems for some linear EPCA in partial derivatives are considered and the behavior of their solutions studied. The results are also extended to equations with positive definite operators in Hilbert spaces.

\section{BOUNDARY VALUE PROBLEMS.}

The equation

$$
u_{t}=a^{2} u_{x x}-b\left(u-u_{0}\right)
$$

describes heat $f l o w$ in a rod with both diffusion $a^{2} u_{x x}$ along the rod and heat loss (or gain) across the lateral sides of the rod. Heat loss $(b>0)$ or gain $(b<0)$ is proportional to the difference between the temperature $u(x, t)$ of the rod and $u_{0}$ of the surrounding medium.

In chemistry where $u$ may stand for concentration, the above equation says that the rate of change $u_{t}$ of the substance is due both to the diffusion $a^{2} u_{x x}$ (in the $x$-direction) and to the fact that the subtance is being created $(b<0)$ or destroyed $(b>0)$ by a chemical reaction proportional to the difference between two concentrations $u$ and $u_{0}[6]$. We may change $u-u_{0}$ to $u$ and consider the equation

$$
u_{t}=a^{2} u_{x x}-b u
$$

Measuring the lateral heat change (or substance change due to a chemical reaction) at discrete moments of time leads to an equation with piecewise constant argument

$$
\begin{aligned}
& u_{t}(x, t)=a^{2} u_{x x}(x, t)-b u(x, n h), \\
& t \in[n h,(n+1) h], \quad n=0,1, \ldots .
\end{aligned}
$$

where $h>0$ is some constant. This equation can be written in the form

$$
u_{t}(x, t)=a^{2} u_{x x}(x, t)-b u(x,[t / h] h),
$$

where $[\cdot]$ designates the greatest integer function. Ordinary differential equations with arguments $[t],[t-n],[t+n]$ have been investigated in $[1-4]$, with $[t+1 / 2]$ in $[5]$, and with $[t / h] h$ in $[7,8]$. Furthermore, EPCA have been used recently in [8] to approximate solu- 
tions of equations with continuous delay.

The diffusion-convection equation

$$
u_{t}=a^{2} u_{x x}-r u_{x}
$$

describes, for instance, the concentration $u(x, t)$ of a pollutant carried along in a stream moving with velocity $r$. The term $a^{2} u_{x x}$ is the diffusion contribution and $-r u_{x}$ is the convection component. If the convection part is measured at discrete times $\mathrm{nh}$, the process results in the equation

$$
u_{t}(x, t)=a^{2} u_{x x}(x, t)-r u_{x}(x,[t / h] h) .
$$

We consider the boundary value problem (BVP) consisting of the equation

$$
\frac{\partial u(x, t)}{\partial t}+p\left[\frac{\partial}{\partial x}\right] u(x, t)=Q\left[\frac{\partial}{\partial x}\right] u(x,[t / h] h),
$$

where $P$ and $Q$ are polynomials of the highest degree $m$ with coefficients that may depend only on $x$, the boundary conditions

$$
\begin{gathered}
L_{j} u=\sum_{k=1}^{m}\left[M_{j k} u^{(k-1)}(0)+N_{j k} u^{(k-1)}(1)\right]=0, \\
\left(M_{j k} \text { and } N_{j k} \text { are constants, } j=1, \ldots, m\right)
\end{gathered}
$$

and the initial condition

$$
u(x, 0)=u_{0}(x)
$$

Here $[\cdot]$ designates the greatest integer function, $(x, t) \in[0,1] x[0, \infty)$, and $\mathrm{h}=$ const $>0$. Equations $(2.4)$ will be written briefly as

$$
\mathrm{Lu}=0 \text {. }
$$

DEFINITION 2.1. A function $u(x, t)$ is called a solution of the above BVP if it satisfies the conditions: (i) $u(x, t)$ is continuous in $G=[0,1] x[0, \infty) ;(i i) \partial u / \partial t$ and $\partial^{k} \mathrm{u} / \partial x^{k}(k=0,1 \ldots, m)$ exist and are continuous in $G$, with the possible exception of the points $(x, n h)$, where one-sided derivatives exist $(n=0,1,2 \ldots)$; (iii) $u(x, t)$ satisfies equation (2.3) in $G$, with the possible exception of the points $(\mathrm{x}, \mathrm{nh})$, and conditions $(2.4)-(2.5)$.

Let $u_{n}(x, t)$ be the solution of the given problem on the interval $n h \leq t<(n+1) h$, then

$$
\partial u_{n}(x, t) / \partial t+P u_{n}(x, t)=Q u_{n}(x),
$$


where

$$
u_{n}(x)=u_{n}(x, n h)
$$

Write

$$
u_{n}(x, t)=w_{n}(x, t)+v_{n}(x),
$$

which gives the equation

$$
\partial w_{n} / \partial t+P w_{n}+P v_{n}(x)=Q u_{n}(x) .
$$

and require that

$$
\begin{gathered}
\partial w_{n} / \partial t+P w_{n}=0, \\
P v_{n}(x)=Q u_{n}(x) .
\end{gathered}
$$

Assuming both $w_{n}$ and $v_{n}$ satisfy $\left(2.4^{\prime}\right)$ leads to an ordinary BVP (2.8)$\left(2.4^{\prime}\right)$, whose solution is denoted by

$$
v_{n}(x)=P^{-1} Q u_{n}(x),
$$

and to BVP $(2.7)-\left(2.4^{\prime}\right)$, whose solution is sought in the form

$$
w_{n}(x, t)=x(x) T_{n}(t) \text {. }
$$

Separation of variables produces the $O D E$

with a solution

$$
T_{n}^{\prime}+\lambda T_{n}=0
$$

$$
T_{n}(t)=e^{-\lambda(t-n h)},
$$

and the BVP

$$
P(d / d x) X-\lambda X=0, \quad L X=0
$$

where $L$ is defined in (2.4) and (2.4'). If BVP (2.10) has an infinite countable set of eigenvalues $\lambda_{j}$ and corresponding eigenfunctions $x_{j}(x) \in C^{m}[0,1]$, then the series

$$
w_{n}(x, t)=\sum_{j=1}^{\infty} c_{n j} e^{-\lambda j(t-n h)} x_{j}(x), c_{n j}=\text { const }
$$

represents a formal solution of problem (2.7)-(2.4') and

$$
u_{n}(x, t)=\sum_{j=1}^{\infty} c_{n j} e^{-\lambda j(t-n h)} x_{j}(x)+P^{-1} Q u_{n}(x)
$$

is a formal solution of $(2.3)-(2.4)$. At $t=n h$ we have

$$
u_{n}(x)=\sum_{j=1}^{\infty} c_{n j} x_{j}(x)+P^{-1} Q u_{n}(x) .
$$

Therefore, assuming the sequence $\left\{x_{j}\right\}$ is complete and orthonormal in 
$c^{m}[0,1]$ yields for the coefficients $c_{n j}$ the formula

$$
\begin{gathered}
c_{n j}=\int_{0}^{1} x_{j}(x)\left(I-P^{-1} Q\right) u_{n}(x) d x, \\
(n=0,1,2 \ldots) .
\end{gathered}
$$

Substituting the initial function $u_{0}(x) \in C^{m}[0,1]$ in $(2.13)$ produces the coefficients $C_{0 j}$, and putting them together with $u_{0}(x)$ in (2.11) as $n=0$ gives the solution $u_{0}(x, t)$ of BVP $(2.3),(2.4),(2.5)$ on the interval $0 \leq t \leq h$. Since $u_{0}(x, h)=u_{1}(x, h)=u_{1}(x)$, we can find from (2.13) the numbers $c_{1 j}$ and then substitute them along with $u_{1}(x)$ in (2.11), to obtain the solution $u_{1}(x, t)$ on $h \leq t \leq 2 h$. This method of steps allows to extend the solution to any interval $n h \leq t \leq(n+1) h$. Furthermore, continuity of the solution $u(x, t)$ implies

$$
u_{n}(x,(n+1) h)=u_{n+1}(x,(n+1) h)=u_{n+1}(x),
$$

hence, at $t=(n+1) h$ we get from (2.11) the recursion relations

$$
u_{n+1}(x)=\sum_{j=1}^{\infty} c_{n j} e^{-\lambda j h} x_{j}(x)+P^{-1} Q u_{n}(x) \text {. }
$$

Therefore,

$$
u_{n+1}(x)=u_{n}(x)-\sum_{j=1}^{\infty} c_{n j}\left(1-e^{-\lambda} j^{h}\right) x_{j}(x)
$$

and

$$
\left(I-P^{-1} Q\right) u_{n+1}(x)=\left(I-P^{-1} Q\right) u_{n}(x)-\sum_{j=1}^{\infty} c_{n j}\left(1-e^{-\lambda} j^{h}\right)\left(I-P^{-1} Q\right) x_{j}(x) .
$$

Multiplying by $x_{k}(x)$ and integrating between 0 and 1 yields the recursion formulas

$$
c_{n+1, k}=c_{n k}-\sum_{j=1}^{\infty} c_{n j}\left(1-e^{-\lambda j h}\right) x_{j k}
$$

where

$$
x_{j k}=\int_{0}^{1} x_{k}(x)\left(I-P^{-1} Q\right) x_{j}(x) d x .
$$

THEOREM 2.1. Formula (2.11), with coefficients $C_{n j}$ and functions $u_{n}(x)$ defined by recursion relations (2.13) and (2.14), represents a formal solution of BVP $(2.3),(2.4),(2.5)$ in $[0,1] \times[n h,(n+1) h]$, for $n=0,1, \ldots$, if BVP $(2.10)$ has a countable number of eigenvalues $\lambda_{j}$ and a complete orthonormal set of eigenfunctions $x_{j}(x) \in C^{m}[0,1]$ and the 
initial function $u_{0}(x) \in C^{m}[0,1]$ satisfies $(2.4)$.

A different method can be used if we look for a solution with continuous derivatives $a^{2} \mathrm{u} / \partial \mathrm{t}^{2}$ and $a^{\mathrm{k}+1} \mathrm{u} / \partial \mathrm{t} \partial \mathrm{x}^{\mathrm{k}}(\mathrm{k}=0,1 \ldots, \mathrm{m})$ for $t \in(n h,(n+1) h)$. In this case we differentiate $(2.6)$ with respect to $t$ and obtain the equation

$$
\partial y_{n} / \partial t+P(\partial / \partial x) y_{n}=0, \quad y_{n}=\partial u_{n} / \partial t,
$$

whose solution is sought in form (2.9). Again, separation of variables produces $T_{n}(t)$ and BVP $(2.10)$. Integrating the solution

$$
y_{n}(x, t)=\sum_{j=1}^{\infty} B_{n j} e^{-\lambda j(t-n h)} x_{j}(x)
$$

between $\mathrm{nh}$ and $\mathrm{t}$ gives

$$
u_{n}(x, t)=u_{n}(x)+\sum_{j=1}^{\infty} B_{n j}\left(1-e^{-\lambda j(t-n h)}\right) x_{j}(x) / \lambda_{j} .
$$

Continuity of the solution at $t=(n+1) h$ implies

$$
u_{n+1}(x)=u_{n}(x)+\sum_{j=1}^{\infty} B_{n j}\left(1-e^{-\lambda j h}\right) x_{j}(x) / \lambda_{j} .
$$

From (2.6) and (2.15) at $t=\mathrm{nh}$ we have

$$
\begin{aligned}
& y_{n}(x, n h)=(Q-P) u_{n}(x), \\
& y_{n}(x, n h)=\sum_{j=1}^{\infty} B_{n j} x_{j}(x),
\end{aligned}
$$

and consequently,

$$
B_{n j}=\int_{0}^{1} x_{j}(x)(Q-P) u_{n}(x) d x .
$$

THEOREM 2.2. Series (2.16), with coefficients $B_{n j}$ and functions $u_{n}(x)$ defined by $(2.17)$ and $(2.18)$, formally represents a solution of BVP $(2.3),(2.4),(2.5)$ whose derivatives $\partial u_{n} / \partial t, \partial u_{n} / \partial x^{k}(k=0,1, \ldots m)$ are continuous in $[0,1] \times[n h,(n+1) h]$ and $\partial^{2} u_{n} / \partial t^{2}, \partial^{k+1} u_{n} / \partial t \partial x^{k}$ are continuous in $[0,1] \times(n h,(n+1) h)$ if, in addition to the other conditions of Theorem 2.1, the initial function $u_{0}(x)$ and $(Q-P) u_{0}(x)$ satisfy $(2.4)$.

The solution $u_{n}(x, t)$ of the nonhomogeneous equation 


$$
\frac{\partial u(x, t)}{\partial t}+P\left[\frac{\partial}{\partial x}\right] u(x, t)=Q\left[\frac{\partial}{\partial x}\right] u(x,[t / h] h)+f(x, t)
$$

on $\mathrm{nh} \leq t<(n+1) h$ is also sought in the form

$$
u_{n}(x, t)=\sum_{j=1}^{\infty} x_{j}(x) T_{n j}(t),
$$

where $x_{j}(x)$ are the eigenfunctions of the operator P. Upon multiplyind (2.19) by $x_{k}(x)$, then integrating between 0 and 1 and changing $k$ to $j$, we obtain

$$
\begin{gathered}
T_{n j}^{\prime}(t)+\lambda_{j} T_{n j}(t)=q_{n j}+f_{j}(t), \\
q_{n j}=\int_{0}^{1} x_{j}(x) Q(d / d x) u_{n}(x) d x, \\
f_{j}(t)=\int_{0}^{1} x_{j}(x) f(x, t) d x,
\end{gathered}
$$

whence

$$
\begin{aligned}
& T_{n j}(t)=\left(T_{n j}(n h)-\lambda_{j}^{-1} q_{n j}\right) e^{-\lambda j(t-n h)} \\
&+\lambda_{j}^{-1} q_{n j}+\int_{n h}^{t} e^{-\lambda} j(t-s) f_{j}(s) d s, \\
&(n h \leq t \leq(n+1) h) \\
& T_{n j}(n h)=\int_{0}^{1} u_{n}(x) x_{j}(x) d x,
\end{aligned}
$$

that is,

$$
\begin{aligned}
T_{n j}(t)= & {\left[\int_{0}^{1} x_{j}(x)\left(I-\lambda_{j}^{-1} Q\right) u_{n}(x) d x\right] e^{-\lambda j(t-n h)}+\lambda_{j}^{-1} \int_{0}^{1} x_{j}(x) Q u_{n}(x) d x } \\
& +\int_{n h}^{t} e^{-\lambda j(t-s)} f_{j}(s) d s .
\end{aligned}
$$

The principal role of the operator $\mathrm{P}$ emerges from the above three methods of constructing the solution. Let

$$
P y=\sum_{j=0}^{m} p_{j} y^{(m-j)},
$$

where $p_{j}$ are real-valued functions of classes $c^{m-j}$ on $0 \leq x \leq 1$ and $p_{0}(x) \neq 0$ on $[0,1]$. Assuming $c^{m}[0,1]$ is embedded in $L^{2}[0,1]$ with the inner product

$$
(y, z)=\int_{0}^{1} y(x) z(x) d x,
$$

BVP (2.10) is called self-adjoint if

$$
(P y, z)=(y, P z),
$$


for all $y, z \in C^{m}[0,1]$ that satisfy the boundary conditions

$$
\mathrm{Ly}=\mathrm{Lz}=0 \text {. }
$$

If BVP (2.10) is self-adjoint, then all its eigenvalues are real and form at most a countable set without finite limit points. The eigenfunctions corresponding to different eigenvalues are orthogonal.

THEOREM 2.3 . BVP $(2.3),(2.4),(2.5)$ has a solution in

$[0,1] \times[n h,(n+1) h]$, for each $n=0,1, \ldots$, given by formula (2.11) if the following hypotheses hold true.

(i) BVP (2.10) is self-adjoint, all its eigenvalues $\lambda_{j}$ are positive.

(ii) For each $\lambda_{j}$, the roots of the equation $P(z)-\lambda_{j}=0$ have non-positive real parts.

(iii) The initial function $u_{0}(x) \in C^{m}[0,1]$ satisfies $(2.4)$.

PROOF. According to (2.8), we find the solution $v_{0}(x)=P^{-1} Q u_{0}(x)$ of the equation $\mathrm{Pv}_{0}(\mathrm{x})=\mathrm{Qu} \mathrm{u}_{0}(\mathrm{x})$ satisfying the boundary conditions $\mathrm{Lv}_{0}=0$. Then the difference $u_{0}(x)-P^{-1} Q u_{0}(x) \in C^{m}[0,1]$ satisfies $\left(2.4^{\prime}\right)$, and therefore we conclude from (2.12) that the Fourier series $\sum c_{0 j} x_{j}(x)$ converges to it absolutely and uniformly on $[0,1]$, where $\left\{x_{j}(x)\right\}$ is the set of the orthonormal eigenfunctions of (2.10). since $\lambda_{j}>0$, the series in $(2.11)$ also converges absolutely and uniformly on $[0,1] \times[0, h]$. Furthermore, the same is true on $[0,1]$ for the series in (2.14) at $n=0$, and $u_{1}(x)$ satisfies (2.4). Hence, $u_{1}(x)$ should be used now to find the solution $v_{1}(x)=P^{-1} Q u_{1}(x)$ of the equation $P v_{1}(x)$ $=Q u_{1}(x)$ satisfying $L_{1}=0$, then to calculate the coefficients $C_{1 j}$ by (2.13) and the solution $u_{1}(x, t)$ of the given BVP on $[0,1] x[h, 2 h]$, according to (2.11). This procedure can be continued successively to construct the solution $u_{n}(x, t)$ for any $n \geq 0$. From (2.12) we conclude that all $u_{n}(x)$ satisfy $\left(2.4^{\prime}\right)$. Differentiating (2.11) term by term 
with respect to $t$ produces a series which converges to $\partial u_{n} / \partial t$ uniformly on $[0,1] \times[n h+\delta,(n+1) h]$, for sufficienty small $\delta>0$, since $\lambda_{j}>0$. Furthermore, it follows from (2.13) that

$$
C_{n j}=\lambda_{j}^{-1} \int_{0}^{1}\left(P X_{j}\right)\left(I-P^{-1} Q\right) u_{n}(x) d x
$$

and since $x_{j}(x), u_{n}(x)$, and $P^{-1} Q u_{n}(x)$ satisfy $\left(2.4^{\prime}\right)$, then

$$
C_{n j}=\lambda_{j}^{-1} \int_{0}^{1} x_{j}(x)(P-Q) u_{n}(x) d x \text {. }
$$

Hence,

$$
\left|c_{n j}\right| \leq \lambda_{j}^{-1}\left[\int_{0}^{1} x_{j}^{2} d x\right]^{1 / 2}\left[\int_{0}^{1}\left(P u_{n}-Q u_{n}\right)^{2} d x\right]^{1 / 2} \leq c_{n} \lambda^{-1} .
$$

Let $p_{0}(x)=1$ and $\lambda=\rho^{m}$, then in any domain $T$ of the complex $\rho-p l a n e$ the equation

$$
P(d / d x) y-\lambda y=0
$$

has $m$ linearly independent solutions $y_{1}, \ldots y_{m}$ which are regular with respect to $\rho \in T$, for sufficiently large $|\rho|$, and satisfy the relations

$$
\begin{gathered}
y_{k}^{(r-1)}(x)=\rho^{r-1} e^{\rho \omega} k^{x}\left[\omega_{k}^{r-1}+o\left(\rho^{-1}\right)\right], \\
(k, r=1, \ldots, m)
\end{gathered}
$$

where $\omega_{1}, \ldots, \omega_{m}$ are the different m-order roots of unity [9]. Therefore, by virtue of condition (ii) and estimates (2.22), differentiating series $(2.11)$ term by term $r$ times $(r=1, \ldots, m)$ with respect to $x$ produces series that converge iniformly on $[0,1] \times[n h+\delta,(n+1) h]$, for sufficiently small $\delta$ and large $\lambda_{j}$. Letting $t=(n+1) h$ in each of these series and taking into account (2.14) shows that $u_{n+1}(x) \in C^{m}[0,1]$ if $u_{n}(x) \in C^{m}[0,1]$. By virtue of (iii), the proof is complete.

REMARK 1. We assumed in this theorem that $p_{0}(x)=1$, where $p_{0}(x)$ is the leading coefficient of the operator $P(d / d x)$. If $p_{0}=$ const $\neq 1$, then dividing the equation $\mathrm{Py}-\lambda y=0$ by $\mathrm{p}_{0}$ produces an equation whose leading coefficient is 1 . If $p_{0}(x) \neq$ const on $[0,1]$ and retains 
its sign, then we may assume $p_{0}(x)>0$ and use the substitution [9]

$$
\mathrm{x}_{1}=\int_{0}^{\mathrm{x}} \mathrm{p}_{0}^{-1 / \mathrm{m}}(\mathrm{s}) \mathrm{ds} / \int_{0}^{1} \mathrm{p}_{0}^{-1 / \mathrm{m}}(\mathrm{s}) \mathrm{ds},
$$

to reduce the above equation to a new one in the interval $0 \leq x_{1} \leq 1$, with a constant leading coefficient.

REMARK 2. The Fourier coefficients used in the above three methods of solving BVP (2.3),(2.4),(2.5) are closely interrelated. Indeed, differentiating (2.11) with respect to $t$ and comparing with (2.15) shows the $B_{n j}=-\lambda_{j} C_{n j}$. Furthermore, comparing (2.11) with $(2.20)$ and (2.13) with (2.21), we have to prove that

$$
\int_{0}^{1} \lambda_{j}^{-1} x_{j}(x) Q u_{n}(x) d x=\int_{0}^{1} x_{j}(x) P^{-1} Q u_{n}(x) d x .
$$

since $P^{-1} Q u_{n}(x)$ satisfies $(2.4)$, then

$$
\mathrm{P}^{-1} \mathrm{Qu} \mathrm{n}_{\mathrm{n}}(\mathrm{x})=\sum_{\mathrm{k}=1}^{\infty} \mathrm{x}_{\mathrm{k}}(\mathrm{x}) \int_{0}^{1} \mathrm{x}_{\mathrm{k}}(\mathrm{x}) \mathrm{P}^{-1} \mathrm{Qu} \mathrm{u}_{\mathrm{n}}(\mathrm{x}) \mathrm{dx},
$$

and applying the operator $P$ to this equation yields

$$
Q u_{n}(x)=\sum_{k=1}^{\infty} \lambda_{k} X_{k}(x) \int_{0}^{1} x_{k}(x) P^{-1} Q u_{n}(x) d x .
$$

It remains to multiply this expansion by $x_{j}(x)$ and to integrate between 0 and 1.

EXAMPLE 2.1. The solution $u_{n}(x, t)$ of equation $(2.1)$ in

$[0,1] \times[n h,(n+1) h]$, with the boundary conditions $u_{n}(0, t)=u_{n}(1, t)=0$ and initial condition $u_{n}(x, n h)=u_{n}(x)$ is sought in form $(2.20)$. Separation of variables produces

$$
x_{j}(x)=\sqrt{2} \sin (\pi j x), T_{n j}^{\prime}(t)+a^{2} \pi^{2} j^{2} T_{n j}(t)=-b T_{n j}(n h),
$$

whence

$$
T_{n j}(t)=c_{n j} e^{-a^{2} \pi^{2} j^{2}(t-n h)}-\frac{b}{a^{2} \pi^{2} j^{2}} T_{n j}(n h) .
$$

We put $t=n h$ in this equation and get

that is,

$$
c_{n j}=\left[1+\frac{b}{a^{2} \pi^{2} j^{2}}\right] T_{n j}(n h),
$$

$$
T_{n j}(t)=E_{j}(t-n h) T_{n j}(n h),
$$


where

$$
E_{j}(t)=e^{-a^{2} \pi^{2} j^{2} t}-\left|1-e^{-a^{2} \pi^{2} j^{2} t}\right| \frac{b}{a^{2} \pi^{2} j^{2}} .
$$

At $t=(n+1) h$ we have

$$
T_{n j}((n+1) h)=E_{j}(h) T_{n j}(n h)
$$

and since

$$
T_{n j}((n+1) h)=T_{n+1, j}((n+1) h),
$$

then

$$
T_{n+1, j}((n+1) h)=E_{j}(h) T_{n j}(n h)
$$

and

$$
T_{n j}(n h)=E_{j}^{n}(h) T_{0 j}(0) \text {. }
$$

Therefore,

$$
T_{n j}(t)=E_{j}(t-n h) E_{j}^{n}(h) T_{0 j}(0)
$$

and

$$
u_{n}(x, t)=\sum_{j=1}^{\infty} \sqrt{2} E_{j}^{n}(h) T_{0 j}(0) E_{j}(t-n h) \sin (\pi j x) .
$$

Putting $t=0, \mathrm{n}=0$ gives

$$
u_{0}(x)=\sum_{j=1}^{\infty} T_{0 j}(0) \sqrt{2} \sin (\pi j x) d x
$$

and

$$
T_{0 j}(0)=\sqrt{2} \int_{0}^{1} u_{0}(x) \sin (\pi j x) d x .
$$

If $\left|E_{j}(h)\right|<1$, then solution (2.24) decays exponentially as $t \rightarrow \infty$, uniformly with respect to $\mathrm{x}$. From (2.23) it follows that this is true if

$$
-a^{2} \pi^{2}<b<a^{2} \pi^{2}\left[e^{a^{2} \pi^{2} h}+1\right] /\left[e^{a^{2} \pi^{2} h}-1\right] .
$$

Furthermore, from the equations

$$
T_{n j}(n h)=E_{j}^{n}(h) T_{0 j}(0), T_{n j}((n+1) h)=E_{j}^{n+1}(h) T_{0 j}(0)
$$

we see that $T_{n j}(n h) T_{n j}((n+1) h)<0$ if $E_{j}(h)<0$. The latter inequality holds true if

$$
b>a^{2} \pi^{2} /\left[e^{a^{2} \pi^{2} h}-1\right] .
$$


Hence, under condition (2.25), each function $T_{n j}(t)(j=1,2, \ldots)$ has a zero in the interval $[\mathrm{nh},(n+1) h]$, in sharp contrast to the functions $T_{j}(t)$ in the Fourier expansion for the solution of the equation $u_{t}=a^{2} u_{x x}$ - bu without time delay. Moreover, the inequality $E_{j}(h)<0$ takes place for sufficiently large $j$ and any $b>0$. Therefore, for $b>0$ and sufficiently large $j$, the functions $T_{n j}(t)$ are oscillatory. EXAMPLE 2.2. Equation (2.2) on $n h \leq t<(n+1)$ h becomes

$$
\partial u_{n}(x, t) / \partial t=a^{2} \partial^{2} u_{n}(x, t) / \partial x^{2}-r u_{n}^{\prime}(x),
$$

and we differentiate the latter with respect to $t$ to obtain the equation

$$
\partial y_{n} / \partial t=a^{2} \partial^{2} y_{n} / \partial x^{2}, \quad y_{n}=\partial u_{n} / \partial t,
$$

whose solution is sought in form (2.9). Separation of variables leads to the equations

$$
x^{\prime \prime}(x)+\lambda x(x)=0, T_{n}^{\prime}(t)+a^{2} \lambda T_{n}(t)=0,
$$

and posing the boundary conditions $u_{n}(0, t)=u_{n}(1, t)=0$ gives $\lambda_{j}=j^{2} \pi^{2}$ and

$$
y_{n}(x, t)=\sum_{j=1}^{\infty} \sqrt{2} T_{n j}(n h) e^{-a^{2} \pi^{2} j^{2}(t-n h)} \sin (\pi j x) .
$$

Since

$$
y_{n}(x, n h)=a^{2} u_{n}^{\prime \prime}(x)-r u_{n}^{\prime}(x), u_{n}(x)=u_{n}(x, n h),
$$

then

$$
a^{2} u_{n}^{\prime \prime}(x)-r u_{n}^{\prime}(x)=\sum_{j=1}^{\infty} \sqrt{2} T_{n j}(n h) \sin (\pi j x)
$$

and

$$
\begin{aligned}
T_{n j}(n h)= & -a^{2} \pi^{2} j^{2} \sqrt{2} \int_{0}^{1} u_{n}(x) \sin (\pi j x) d x \\
& +r \pi j \sqrt{2} \int_{0}^{1} u_{n}(x) \cos (\pi j x) d x .
\end{aligned}
$$

Finally,

$u_{n}(x, t)=u_{n}(x)+\sum_{j=1}^{\infty} \sqrt{2} T_{n j}(n h)\left\{1-e^{-a^{2} \pi^{2} j^{2}(t-n h)}\right] \sin (\pi j x) / a^{2} \pi^{2} j^{2}$.

Given the initial function $u(x, 0)=u_{0}(x)$, we can find the coefficients $T_{0 j}(0)$ and the solution $u_{0}(x, t)$ on $0 \leq t \leq h$. Since $u_{0}(x, h)$ $=u_{1}(x)$, we can calculate the coefficients $T_{1 j}(h)$ and the solution 
$u_{1}(x, t)$ on $h \leq t \leq 2 h$. By the method of steps the solution can be extended to any interval $[\mathrm{nh},(n+1) h]$.

EXAMPLE 2.3. Separation of variables for the equation

$$
\partial u(x, t) / \partial t=a^{2} \partial^{2} u(x, t) / \partial x^{2}-b \partial^{2} u(x,[t]) / \partial x^{2}
$$

with the boundary conditions $u(0, t)=u(1, t)=0$ produces the eigenfunctions $x_{j}(x)=\sqrt{2} \sin (\pi j x)$ and the equation

$$
T^{\prime}(t)+a^{2} \pi^{2} j^{2} T(t)=b \pi^{2} j^{2} T([t]),
$$

which on the interval $n \leq t<n+1$ becomes

$$
T_{n j}^{\prime}(t)+a^{2} \pi^{2} j^{2} T_{n j}(t)=b \pi^{2} j^{2} T_{n j}(n) .
$$

From here,

$$
T_{n j}(t)=F_{j}(t-n) T_{n j}(n),
$$

where

$$
F_{j}(t)=e^{-a^{2} \pi^{2} j^{2} t}+\left[1-e^{-a^{2} \pi^{2} j^{2} t}\right] b / a^{2} .
$$

At $t=n+1$ we have

$$
T_{n j}(n+1)=F_{j}(1) T_{n j}(n)
$$

and since $T_{n j}(n+1)=T_{n+1, j}(n+1)$, then

$$
T_{n+1, j}(n+1)=F_{j}(1) T_{n j}(n)
$$

and

$$
T_{n j}(n)=F_{j}^{n}(1) T_{0 j}(0)
$$

Hence,

$$
u_{n}(x, t)=\sum_{j=1}^{\infty} \sqrt{2} F_{j}^{n}(1) T_{0 j}(0) F_{j}(t-n) \sin (\pi j x),
$$

where

$$
T_{0 j}(0)=\sqrt{2} \int_{0}^{1} u_{0}(x) \sin (\pi j x) d x .
$$

The inequalities

$$
-a^{2}\left[e^{a^{2} \pi^{2}}+1\right] /\left[e^{a^{2} \pi^{2}}-1\right]<b<a^{2}
$$

are equivalent to $\left|F_{j}(1)\right|<1$ and ensure the exponential decay of 
$u_{n}(x, t)$ as $t \rightarrow \infty$, uniformly with respect to $x$. For

$$
b<-a^{2} /\left(e^{a^{2} \pi^{2}}-1\right),
$$

each function $T_{n j}(t)$ has a zero in the interval $[n, n+1]$, which is impossible for the equation $u_{t}=\left(a^{2}-b\right) u_{x x}$.

EXAMPLE 2.4. The equation

$$
\text { iq } \frac{\partial u(x, t)}{\partial t}=-\frac{q^{2}}{2 m_{0}} \frac{\partial^{2} u(x, t)}{\partial x^{2}}+v(x) u(x,[t / h] h)
$$

is a piecewise constant analogue of the one-dimensional schrödinger equation

$$
i q \psi_{t}(x, t)=-q^{2} \psi_{x x}(x, t) / 2 m_{0}+v(x) \psi(x, t) .
$$

If $u(x, t)$ satisfies conditions $(2.4)$ and $(2.5)$, with $m=2$, then separation of variables produces a formal solution

$$
u_{n}(x, t)=\sum_{j=1}^{\infty} c_{n j} e^{-i \lambda j(t-n h) / q_{x_{j}}(x)+P^{-1} Q u_{n}(x),}
$$

for $n h \leq t \leq(n+1) h$. Here $x_{j}(x)$ are the eigenfunctions of the operator $\mathrm{q}^{2}\left(\mathrm{~d}^{2} / \mathrm{dx}^{2}\right) / 2 \mathrm{~m}_{0}$, and $\mathrm{P}^{-1} \mathrm{Qu}_{\mathrm{n}}(\mathrm{x})$ is the solution $\mathrm{v}_{\mathrm{n}}(\mathrm{x})$ of the equa-
tion

$$
q^{2} v_{n}^{\prime \prime}(x)=2 m_{0} v(x) u_{n}(x)
$$

that satisfies (2.4) and $c_{n j}$ are given by (2.13).

The Fourier method can be also used to find weak solutions of BVP $(2.3),(2.4),(2.5)$ and it is easily generalized to similar problems in Hilbert space. First, we remind a few well known definitions. Let $\mathrm{H}$ be a Hilbert space and let $\mathrm{P}$ be a linear operator in $\mathrm{H}$ (additive and homogeneous but, possibly, unbounded) whose domain $D(P)$ is dense in $\mathrm{H}$, that is $\overline{\mathrm{D}(\mathrm{P})}=\mathrm{H}$. The operator $\mathrm{P}$ is called symmetric if $(\mathrm{Pu}, \mathrm{v})$ $=(\mathrm{u}, \mathrm{Pv})$, for any $\mathrm{u}, \mathrm{v} \in \mathrm{D}(\mathrm{P})$. If $\mathrm{P}$ is symmetric, then $(\mathrm{Pu}, \mathrm{v})$ is $a$ symmetric bilinear functional and $(P u, u)$ is a quadratic form. A symmetric operator $P$ is called positive if $(P u, u) \geq 0$ and $(P u, u)=0$ if and only if $u=0$. A symmetric operator $P$ is called positive definite if there exists a constant $\gamma^{2}>0$ such that $(P u, u) \geq \gamma^{2}\|u\|^{2}$. with every positive operator $\mathrm{P}$ a certain Hilbert space $\mathrm{H}_{\mathrm{P}}$ can be associated, which is called the energy space of $P$. It is the completion of $D(P)$, with the inner product $(u, v)_{P}=(P u, v) ; u, v \in D(P)$. This pro- 
duct induces a new norm $\|u\|_{P}=(P u, u)^{1 / 2}, u \in D(P)$, and if $P$ is positive definite, then $\|u\| \leq \gamma^{-1}\|u\|_{P}$. Since $D(P)$ is dense in $H$, it follows by using the latter inequality that the energy space $\mathrm{H}_{\mathrm{P}}$ of a positive definite operator $P$ is dense in the original space $H$.

Assuming $\mathrm{P}$ is positive definite, we may consider the solution $u(x, t)$ of BVP $(2.3),(2.4),(2.5)$ for a fixed $t$ as an element of $H_{P}$. If $D(Q) C H$, then $Q u(x,[t / h] h)$ may be treated as an abstract function $\mathrm{Qu}([t / h] h)$ with the values in $H$. Therefore, the given BVP is reduced to the abstract Cauchy problem

$$
\frac{d u}{d t}+P u=Q u([t / h] h), t>0, u I_{t=0}=u_{0} \in \mathrm{H} .
$$

If (2.26) has a solution, we multiply each term by an arbitrary function $g(t) \in H_{P}$ in the sense of inner product in $H$ and get on $n h \leq t<(n+1) h$ the equation

$$
\left[\frac{d u}{d t}, g\right]+(u, g)_{P}=\left(Q u_{n}, g\right),
$$

where $u_{n}=u(n h)$. Conversely, if $u \in c^{1}((n h,(n+1) h) ; D(P))$ for all integers $n \geq 0$ and satisfies (2.27), then it also satisfies equation (2.26). Indeed, if $u \in D(P)$, then $(u, g)_{P}=(P u, g)$, and (2.27) can be written as

$$
\left[\frac{d u}{d t}+p u-Q u_{n}, g\right]=0, \quad n h \leq t<(n+1) h .
$$

Since $H_{P}$ is dense in $H$, then $u(t)$ is a solution of equation (2.26).

DEFINITION 2.2. An abstract function $u(t):[0, \infty) \rightarrow H$ is called a weak solution of problem (2.26) if it satisfies the conditions: (i) $u(t)$ is continuous for $t \geq 0$ and strongly continuously differentiable for $t>0$, with the possible exception of the points $t=\mathrm{nh}$ where one-sided derivatives exist; (ii) $u(t)$ is continuous for $t>0$ as an abstract function with the values in $\mathrm{H}_{\mathrm{p}}$ and satisfies equation (2.27) on each interval $n h \leq t<(n+1) h$, for any function $g(t):[0, \infty) \rightarrow H_{p}$; (iii)u(t) satisfies initial condition (2.26), that is,

$$
\lim _{t \rightarrow 0}\left\|u(t)-u_{0}\right\|_{H}=0 \text {. }
$$

Clearly, a weak solution $u(t)$ is also an ordinary solution if $u(t) \in D(P)$, for any $t>0$, and $u(x, t) \rightarrow u_{0}(x)$ as $t \rightarrow 0$ not only in 
the norm of $\mathrm{H}$ but uniformly as well. It is said [10] that a symmetric operator $P$ has a discrete spectrum if it has an infinite sequence $\left\{\lambda_{j}\right\}$ of eigenvalues with a single limit point at infinity and a sequence $\left\{x_{j}\right\}$ of eigenfunctions which is complete in $H$. Suppose the operator $P$ in (2.27) is positive definite and has a discrete spectrum and assume existence of a solution $u(t)=u(x, t)$ to equation (2.27) with the condition $u(0)=u_{0}$. On the interval $n h \leq t<(n+1) h$ this solution can be expanded into series (2.20), where $T_{j}(t)=\left(u(t), x_{j}\right)$. To find the coefficents $T_{j}(t)$, we put $g(t)=x_{k}$ in (2.27) and since $x_{k}$ does not depend on $t$, then

$$
\begin{gathered}
{\left[\frac{d u(t)}{d t}, x_{k}\right]=\frac{d}{d t}\left(u(t), x_{k}\right)=T_{k}^{\prime}(t),} \\
\left(u, x_{k}\right)_{p}=\left(P u, x_{k}\right)=\left(u, P x_{k}\right)=\lambda_{k}\left(u, x_{k}\right)=\lambda_{k} T_{k}(t),
\end{gathered}
$$

which again leads to the equation

$$
T_{n j}^{\prime}(t)+\lambda_{j} T_{n j}(t)=\left(Q u_{n}, x_{j}\right)
$$

and to a generalization of (2.21). By selecting a proper space $H, a$ weak solution corresponding to conditions (2.4) can be constructed. The proof of the following theorem is omitted.

THEOREM 2.4. If $P$ and $Q$ are linear operators in a Hilbert space and $\mathrm{P}$ is positive definite with a discrete spectrum, then there exists a unique weak solution of problem (2.26).

ACKNOWLEDGMENT: Research partially supported by U.S. Army Grant DAAL03-89-G-0107.

\section{REFERENCES}

1. COOKE, K. and WIENER, J. Retarded differential equations with piecewise constant delays, J. Math. Anal. Appl.99 (1984), 265-297.

2. COOKE, K. and WIENER, J. Neutral differential equations with piecewise constant arguments, Boll. Unione Mat. Ital. 7, 1-B (1987), 321-346.

3. WIENER, J. and COOKE, K. Oscillations in systems of differential equations with piecewise constant argument, J. Math. Anal. Appl. 137 (1989), 221-239. 
4. SHAH, S.M. and WIENER, J. Advanced differential equations with piecewise constant argument deviations, Internat. J. Math.\& Math. Sci. 6 (1983), 671-703.

5. COOKE, K. and WIENER, J. An equation alternately of retarted and advanced type, proc. Amer. Math. Soc. 99 (1987), 726-732.

6. FARLOW, S.J. Partial Differential Equations for Scientists and Engineers, John Wiley, 1982.

7. COOKE, K. and WIENER, J. Stability regions for linear equations with piecewise continuous delay, Comp. \& Math. with Appls. ILA $(1986), 695-701$.

8. GYÖR, I. On approximation of the solutions of delay differential equations by using piecewise constant arguments, Internat. J. Math. \& Math. Sci. Vol. 14 (1991) 111-126.

9. NAIMARK, M.A. Linear Differential Operators (Russian), Moscow, 1954.

10. MIKHLIN, S.G. Linear Equations in Partial Derivatives (Russian), Moscow, 1977. 


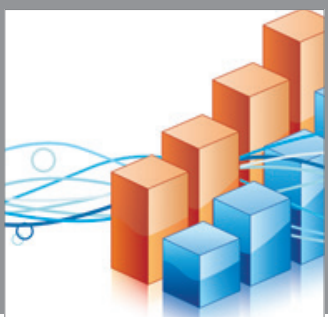

Advances in

Operations Research

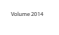

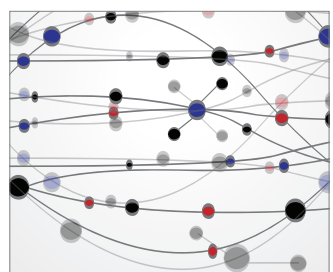

\section{The Scientific} World Journal
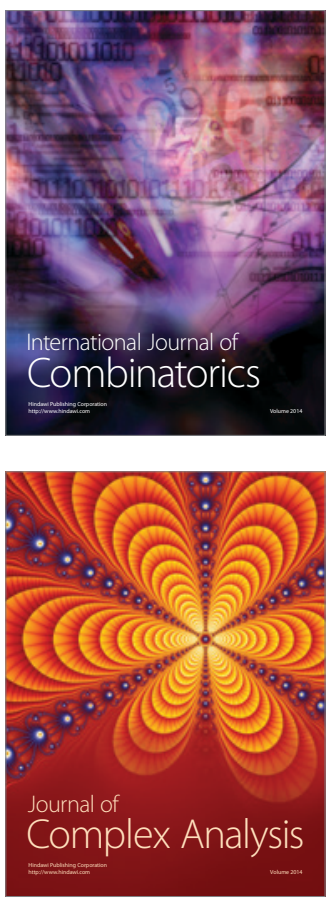

International Journal of

Mathematics and

Mathematical

Sciences
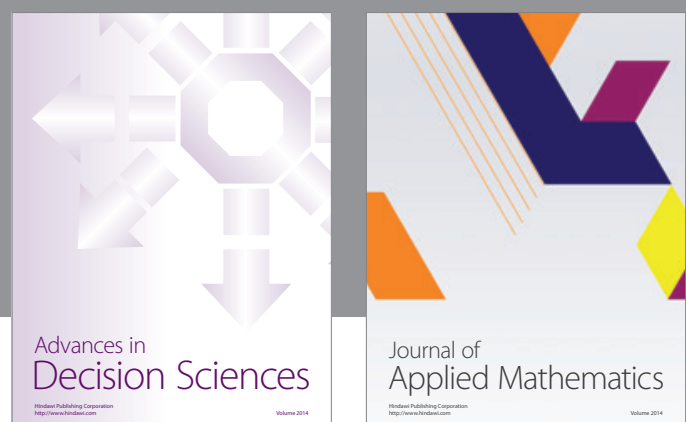

Journal of

Applied Mathematics
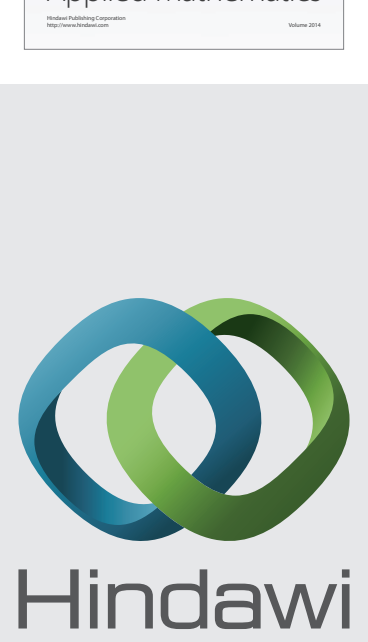

Submit your manuscripts at http://www.hindawi.com
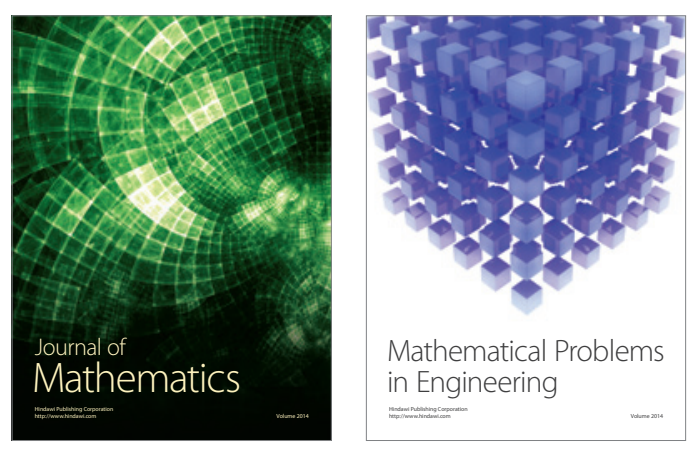

Mathematical Problems in Engineering
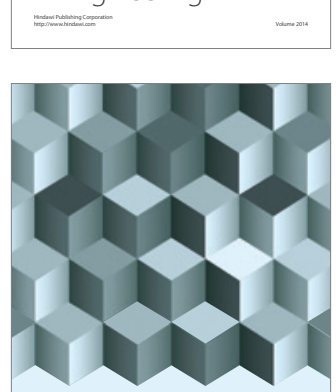

Journal of

Function Spaces
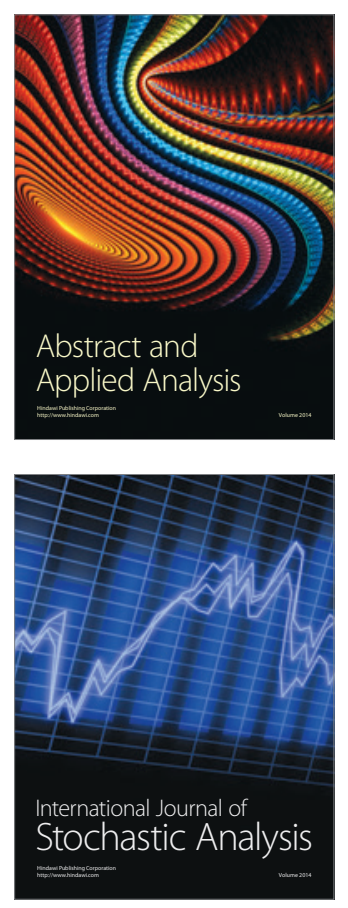

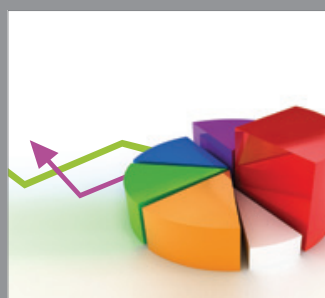

ournal of

Probability and Statistics

Promensencen
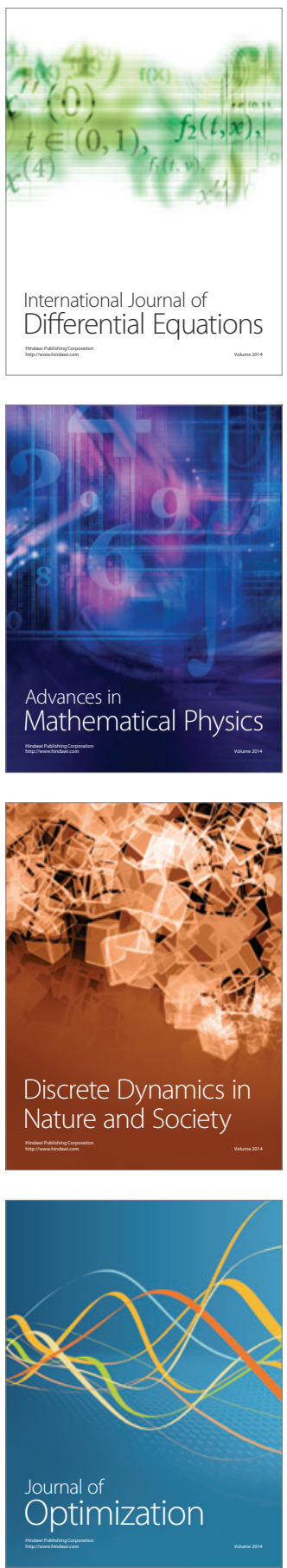